\title{
Risk network structure in the early epidemic phase of HIV transmission in Colorado Springs
}

\author{
J J Potterat, L Phillips-Plummer, S Q Muth, R B Rothenberg, D E Woodhouse, \\ T S Maldonado-Long, H P Zimmerman, J B Muth
}

This study describes the risk network structure of persons with HIV infection during its early epidemic phase in Colorado Springs, USA, using analysis of community-wide HIV/AIDS contact tracing records (sexual and injecting drug partners) from 1985 to 1999. Paired partner information from other STD/HIV programme records was used to augment network connections. Analyses were conducted with and without this supplemental information. The results suggest that a combined dendritic and cyclic structural network pattern is associated with low to moderate HIV propagation in Colorado Springs, and may account for the absence of intense propagation of the virus.

See end of article for authors' affiliations

Correspondence to

Correspondence to:
John J Potterat, STD/HIV Program, El Paso County Department of Health and Environment, 301 South Union Blvd, Colorado Springs, Colorado 80910, USA;

iipotterat@earthlink.net

Accepted

6 December 2001
W th bacterial sexually transmitted diseases (STD), risk network structure has recently been associated with epidemic phase. Empirical evidence suggests that dendritic networks (characterised by linear chains of connections with additional partners connected to each node, but not to each other) maintain the endemic or decline phases of transmission. Cyclic networks, which contain significant numbers of small groups of interconnected people, support the growth phases. ${ }^{1}$ Empirical evidence for this association with human immunodeficiency virus (HIV) infection has not been reported. In this study, we analyse data obtained from 15 years of interviewing HIV positive patients for at-risk partner information to elucidate network patterns and their association with epidemic phase. By uniquely identifying partners named by respondents, personal networks were combined to generate a view of the larger networks in which patients and their partners are embedded. The configuration of risk networks early in Colorado Springs's HIV epidemic was of particular interest in determining the relation of network structure to epidemic phase.

\section{METHODS}

During the early phase of the HIV epidemic in Colorado Springs, the area had a population of 309424 persons (1980 census) - $87.3 \%$ white, 8.1\% Hispanic, 6.2\% African American, and 6.5\% other. Approximately $10 \%$ of the population consisted of active duty military personnel. In Colorado, AIDS has been a reportable condition since 1983, and HIV infection since November 1985; the AIDS/HIV secular trend from 1986 through 1999 appears in fig 1. Although the first case of AIDS in Colorado Springs was interviewed for risk partner information (August 1982, at the request of the Centers for Disease Control and Prevention), routine interviewing of newly diagnosed HIV/AIDS cases for risk partner information did not begin until the Autumn of 1985, a few months after introduction of commercially available HIV antibody tests. Interviewing of all public, private, and military sector cases has been conducted by trained local health department contact tracers continuously since then, with the exception that local military authorities, starting in the spring of 1987, decided to use their own public health staff to conduct interviews of military HIV patients. ${ }^{2}$ HIV interview records remained in military custody, with only identifying information on civilian partners being forwarded to the health department. Thus information linking military index patients with their non-civilian partners from mid-1987 to the present is unavailable for analysis.

Starting in November 1985, all newly diagnosed HIV/AIDS cases reported to the health department were offered contact tracing services. Cases were interviewed for at-risk (sexual and injecting drug use) partners using a flexible period, with special emphasis on current partners (within 12 months preceding diagnosis in the index case). Seeking partners antecedent to this period was attempted if the index patient's exposure or symptomatic history suggested that offering counselling and/or testing to these "historical" partners would be beneficial.

To augment information obtained by formal contact interviewing, health department charts from the HIV Counseling and Testing Center (CTC) were canvassed for linked-pairs information. Clients presenting at our CTC often volunteer names of partners, especially stable partners who simultaneously seek this service; names were recorded in both the client chart and its electronic counterpart. In addition, all routine programme STD (syphilis, gonorrhoea, and chlamydia) contact interviews conducted between 1977 and 1999 ( $\mathrm{n}=32$ 554) were manually canvassed to add sexually linked pairs of men to the HIV contact tracing database.

Local HIV/AIDS surveillance information suggested that most HIV transmission in Colorado Springs occurred from the early to mid-1980s. We therefore divided linked pairs data, based on earliest date of exposure for named partners, into three periods: early (1982-89), middle (1990-94), and late (1995-99). Although the intervals are 


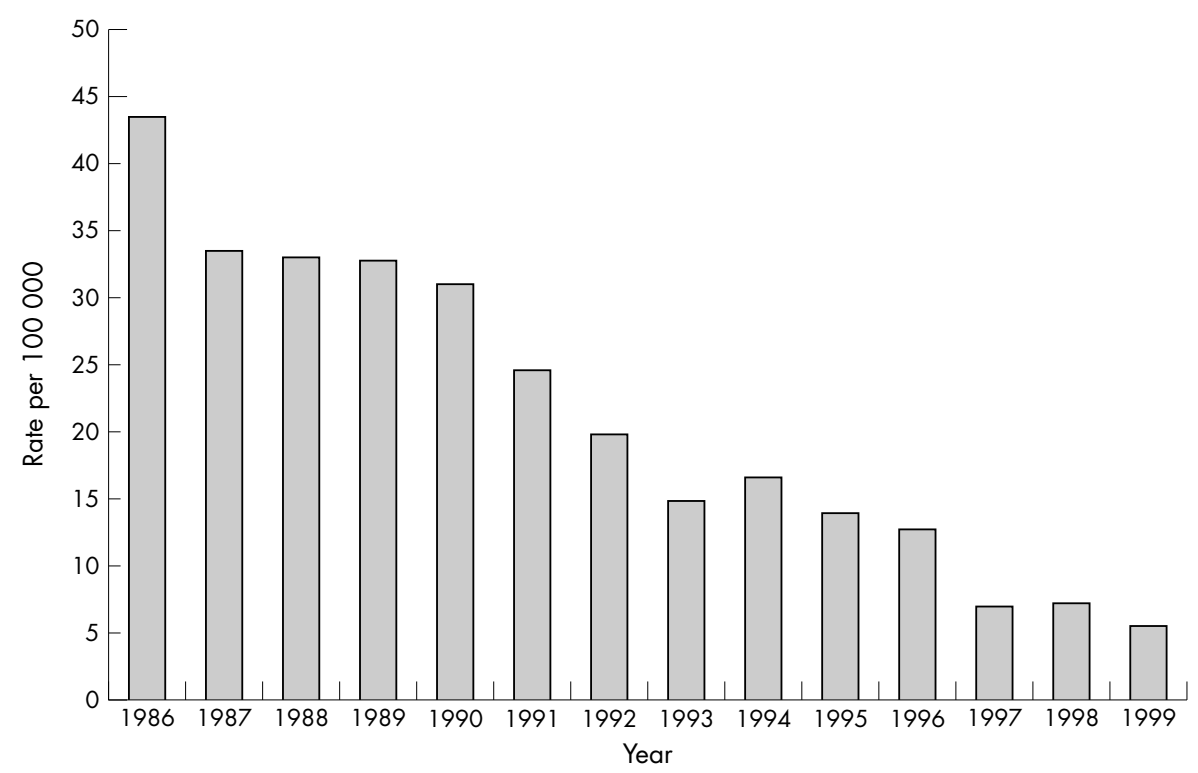

Figure $1 \mathrm{HIV} /$ AIDS case rates $/ 100000$, by year of first diagnosis, Colorado Springs, 1986-99.

unequal, there were few datapoints before availability of HIV antibody tests in mid-1985 (table 1).

This study's focus is on construction of risk networks using known linkages rather than on tracing HIV transmission flow. When known, however, HIV status is illustrated in network figures. Network analysis and visualisation was conducted as previously described.

\section{RESULTS \\ HIV/AIDS cases}

In all, 1323 adult HIV/AIDS cases ( 1168 men and 155 women over the age of 12 years) were reported to our health department between 1982 and 1999. About two thirds (67.5\%) were reported from private sources, one fifth $(21.1 \%)$ from public sources, and one tenth (11.4\%) from the military. Of the 1100 men with HIV/AIDS for whom risk factor information is available, 947 (86\%) were classified as gay/bisexual, including 185 who additionally reported a history of injecting drug use; $133(12.1 \%)$ reported histories of injecting drug use and heterosexuality; $18(1.6 \%)$ were classified as transfusion associated cases; and two $(0.2 \%)$ as heterosexual cases. Of the 147 women with available information, $88(60 \%)$ were classified as injecting drug users; 52 (35.4\%), as heterosexual; and seven $(4.8 \%)$ as transfusion associated cases.

\section{Interviews and named partners}

Of the 1323 cases, 796 (60.2\%) were newly diagnosed locally, and 527 (39.8\%) were new arrivals in Colorado Springs who had been diagnosed and counselled elsewhere. Programmatic focus on interrupting local HIV transmission favoured interviewing locally diagnosed cases. Nearly $70 \%$ of such cases (549 of 796; newly diagnosed cases, table 1) were interviewed, compared with only one quarter of the cases diagnosed elsewhere ( 128 of 527). The highest proportion of interviewed cases occurred during the early period (table 1); there was a pronounced decrease in the proportion of new diagnoses during the 1990s and a corresponding decline in numbers and proportion of cases interviewed for at-risk partner information.

The 677 persons interviewed (580 men and 97 women) nominated 1592 persons (1240 men and 352 women) as partners. Reported partnerships were principally sexual: 1484 sexual exposure only, 46 sex and injecting drug use, and 62 injecting drug use only. Overall, gay/bisexual men were less likely to reveal identifiable partners than heterosexuals, nominating a mean of 2.2 partners per interview $v 2.7$ for heterosexuals ( $\mathrm{p}<0.0001$, Student's $t$ test). Of the 1592 partners, 789 (49.6\%) were HIV positive, 294 (18.5\%) tested negative for antibody to HIV, 465 (29.2\%) were not located, and $44(2.7 \%)$ declined to be tested. Of cases eligible for interview (that is, newly diagnosed), 549 were interviewed and 247 were not ( 114 not located; 47 military cases; 35 counselled rather than interviewed by non-health-department providers; 25 administrative oversights, 13 refusals; and 13 deaths before interview).

\section{Linked partner information}

Of 1933 partners in our dataset, 1592 (82.4\%) were ascertained from HIV contact interviews; 150 (7.8\%) from CTC charts; 144 (7.5\%) from the 293 (0.9\%) STD contact interviews containing identifiable male sexual pairs; 45 $(2.3 \%)$ from exogenous index cases; and two "other". By earliest date of exposure, most $(58.7 \%)$ of the 1592 named partners were assigned to the early period; $27.6 \%$ to the middle period; and $13.6 \%$ to the late period (table 1 ).

\begin{tabular}{|c|c|c|c|c|c|c|c|}
\hline \multirow[b]{2}{*}{$\begin{array}{l}\text { Epidemiological } \\
\text { period }\end{array}$} & \multicolumn{3}{|l|}{ Cases } & \multicolumn{4}{|l|}{ Interviews } \\
\hline & All & $\begin{array}{l}\text { Newly } \\
\text { diagnosed }\end{array}$ & $\begin{array}{l}\text { (Per cent of all } \\
\text { cases) }\end{array}$ & Interviewed & $\begin{array}{l}\text { (Per cent of all } \\
\text { cases) }\end{array}$ & Partners & $\begin{array}{l}\text { (Per cent of total } \\
\text { partners) }\end{array}$ \\
\hline 1982 to 1984 & 6 & 5 & $(66.7)$ & 1 & (16.7) & 1 & $(<1)$ \\
\hline 1985 to 1989 & 465 & 383 & (82.4) & 288 & (61.9) & 934 & (58.7) \\
\hline 1990 to 1994 & 489 & 245 & $(50.1)$ & 252 & (51.5) & 440 & $(27.6)$ \\
\hline 1995 to 1999 & 363 & 164 & (45.2) & 136 & (37.5) & 217 & (13.6) \\
\hline Total & 1323 & 796 & $(60.2)$ & 677 & (51.2) & 1592 & $(100)$ \\
\hline
\end{tabular}


A

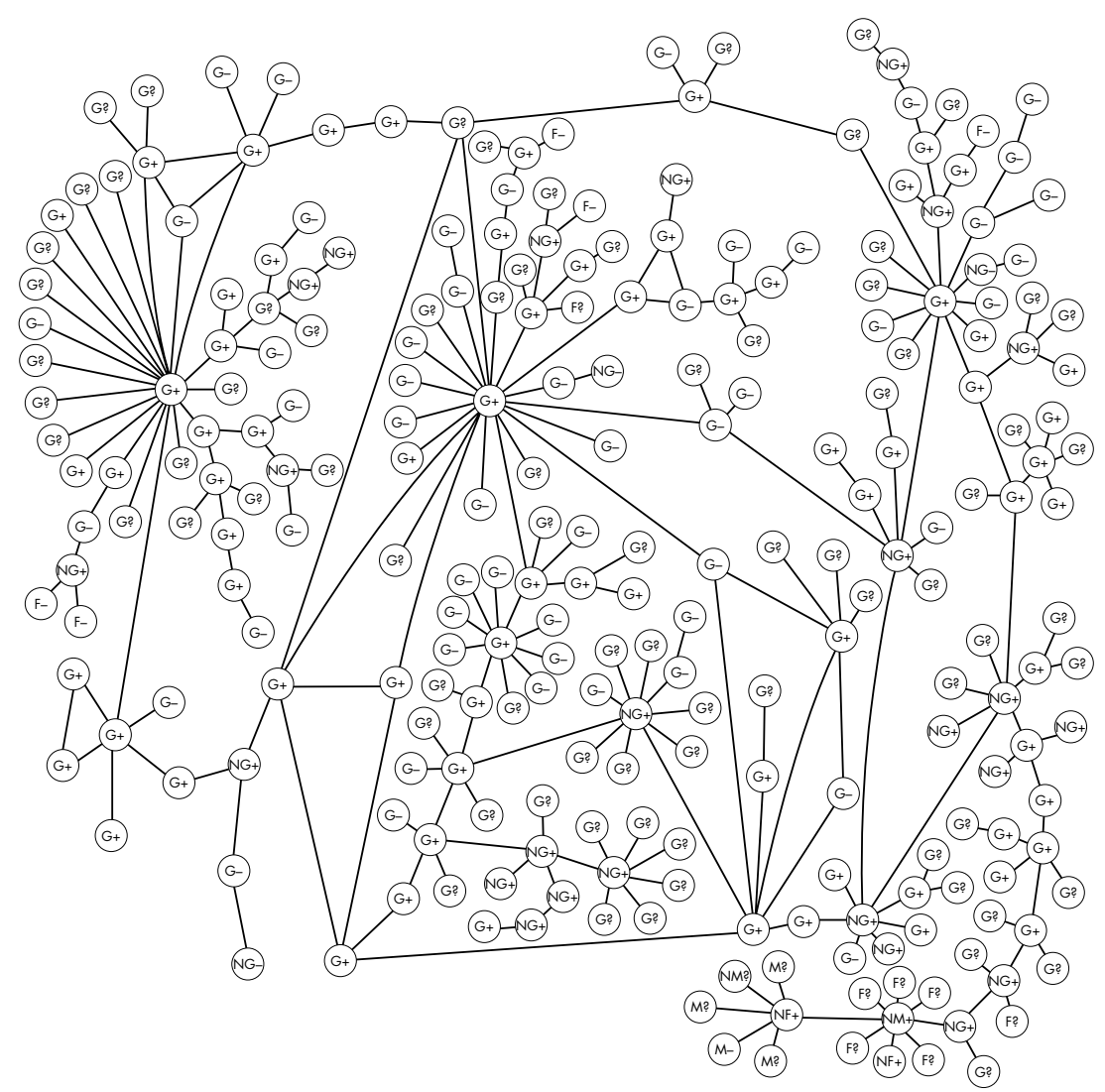

B

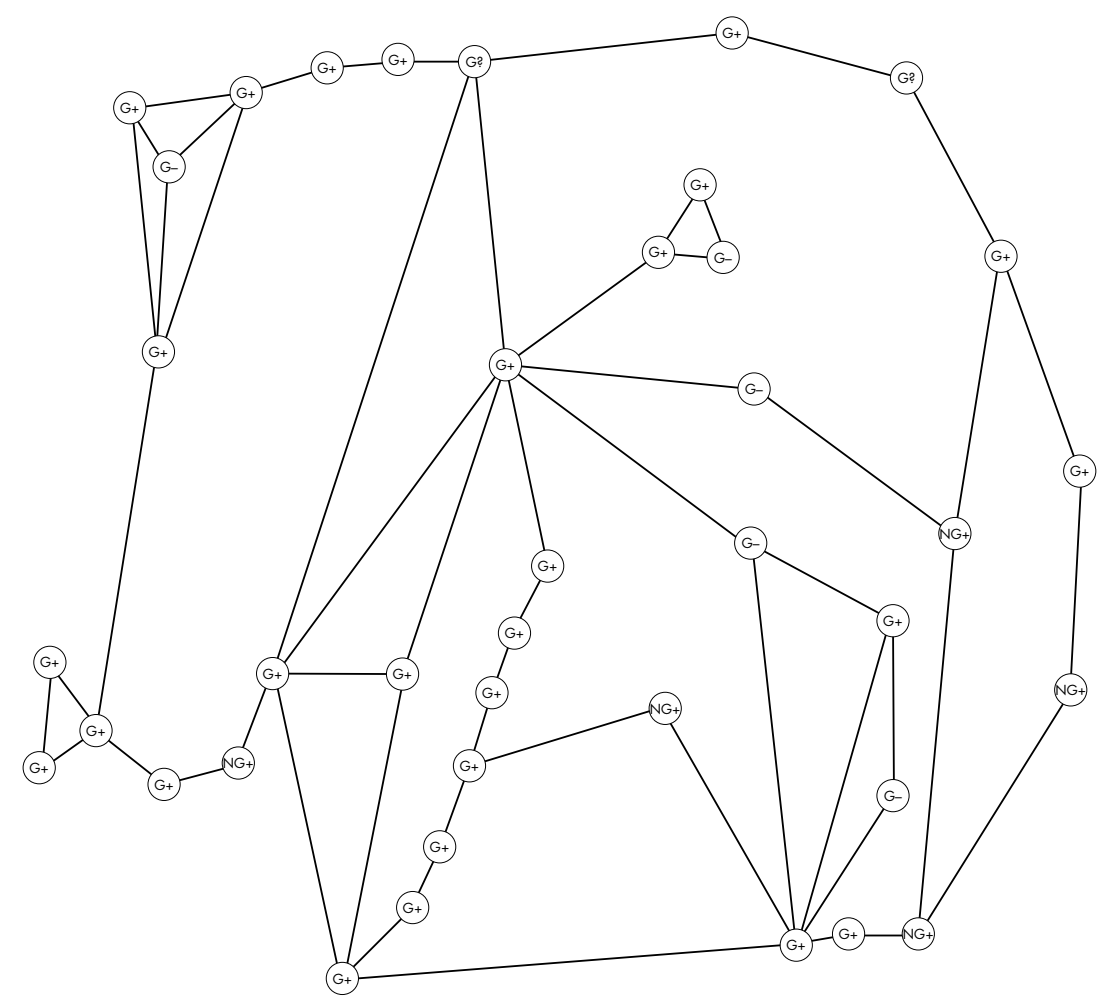

Figure 2 (A) Largest HIV/AIDS connected component, early period (1980s), Colorado Springs ( $\mathrm{n}=$ 250). (B) Largest HIV/AIDS connected component after stepwise graph reduction (removal of branches and termini), early period, Colorado Springs $(n=39)$. Key:

$\mathrm{G}+$, HIV positive gay man $(\mathrm{n}=71)$; G-, HIV negative gay man $(\mathrm{n}=$ 53); G? , gay man, unknown HIV status ( $\mathrm{n}=78$ ); $\mathrm{NG}+$, HIV positive injecting drug using ( $N=$ needles) gay man ( $n=23) ; \mathrm{NG}-$, HIV

negative injecting drug using gay man $(n=4) ; N G$ ?, injecting drug using gay man, unknown HIV status ( $\mathrm{n}=1) ; \mathrm{F}-$, HIV negative woman ( $\mathrm{n}$ = 5); Fe, woman, unknown HIV status ( $\mathrm{n}=7$ ); $\mathrm{NF}+$, injecting drug using HIV positive woman $(\mathrm{n}=1)$; NF?, injecting drug using woman, unknown HIV status $(\mathrm{n}=1)$; $M$ HIV negative heterosexual man $(\mathrm{n}=$ 1); $M$ ? , heterosexual man, unknown HIV status ( $\mathrm{n}=3$ ); $\mathrm{NM+}$, injecting drug using HIV positive man $(\mathrm{n}=$ 1); NM?, injecting drug using man, unknown HIV status $(\mathrm{n}=1)$.

Partner networks: overall observations

Aggregated over the three periods (1980s to 1999) the 1933 partners formed 543 separate components. The largest component contained 324 persons while the second largest had only 28 . Five per cent of components ( 28 of 543 ) had 10 or more connected persons and contained $30 \%$ of persons. The next $36 \%$ of persons occupied the 157 middle sized components (four to nine connected persons each), while $34 \%$ of persons were distributed in the 83 triads and in the 275 dyads. Structurally, the largest component appeared to be evenly balanced between dendritic and cyclic groupings. All smaller components were almost exclusively dendritic, with only a dozen cycles (closed structures) among them. Thus, except for the largest component, our entire cohort of HIV 
partners was fragmented into numerous small dendritic components.

\section{Partner networks: early period}

When divided into early, middle, and late time periods, the overall network configuration retained a similar pattern of fragmentation but the largest component diminished by a factor of 10 or more for the middle and late periods. Only during the early period did the largest connected component remain large $(n=250$, or $77 \%$ of its overall size for all three periods combined), suggesting the presence of sufficient network connectivity to sustain endemic transmission. During this early period, the second largest component contained only 16 persons. Hence we focus on the earliest and largest risk network to generate a picture of early HIV propagation locally.

The stereotypic member of the largest early period component (fig 2A) was a white gay man nearly 30 years old who associated with injecting drug users. Of the 250 persons in that network, $236(94.4 \%)$ were men, of whom 219 were homosexual, and $14(5.6 \%)$ were women. Of the 184 men and 13 women for whom age was known, mean age (at mid-early period) was 29.5 years for men and 25.8 years for women. Of the $194(77.6 \%)$ persons for whom race or ethnicity was known, 171 (88.1\%) were white, 17 (8.8\%) were Hispanic, five (2.6\%) were African American, and one (0.5\%) was an Asian/ Pacific Islander. Of the 250 persons, 32 were known injecting drug users ( 28 gay men, two women, and two heterosexual men).

HIV status was available for 159 persons (63.6\%): 96 HIV positive, 63 HIV negative, and 91 unknown (legend, fig 2A). Notably, 23 (24.5\%) of 94 HIV positive gay men in this network were known injecting drug users compared with four $(7 \%)$ of 57 HIV negative gay men being known injecting drug users, suggesting that HIV may have been more efficiently transmitted through injecting drug use.

Figure 2A depicts the hybrid (dendritic-cyclic) and planar (lacking crossed lines) nature of connections among the 250 persons of the early period network, a pattern suggestive of moderate epidemic transmission. Of the 250 persons, 156 were identified solely by HIV contact interviews, while the remaining 94 persons were identified from the other sources (CTC charts, other interviews, and so on). Removal of these 94 persons did not alter the geometry of this network. That this planar dendritic-cyclic pattern was not only observed in the overall largest component $(\mathrm{n}=324$ persons $)$ and in the largest early period component $(n=250)$, but also in the minimalised (minus the 94 persons from non-interview sources) early period largest component $(n=156$, data not shown) suggests that the observed geometry of our HIV partners risk networks mirrors reality. Note that all heterosexual men and most women appear at the terminus of the longest branch (lower right hand side of fig 2A), bridged by two injecting drug use gay men.

Using a stepwise reduction technique, ${ }^{3}$ branching patterns were systematically removed, leaving only nodes that were cyclically connected (fig 2B). These 39 persons form what one of us (DEW) refers to as "la crème du core"; note that all are gay men and that more than three quarters are HIV positive, including the five who were also injecting drug users. This inner core of transmitting individuals forms ground zero for initial, sustained HIV propagation in Colorado Springs.

\section{DISCUSSION}

Empirical evidence suggests that the "geometry" of networks is related to STD epidemic phase: dendritic risk networks appear to be related to stable or declining endemic settings, ${ }^{4}$ and cyclic risk networks, to accelerating transmission. ${ }^{1}$ Our analysis of the risk networks of HIV/AIDS cases in the same community over time reveals a fragmented pattern, both in the short term (five years) and in the long term ( 15 years). The largest component not only highlights the actors most likely to have played the leading role in endogenous transmission of HIV but also, interestingly, spans the entire period of observation. Most important, this network displays a largely hybrid pattern of dendritic and cyclic subgroups. This hybrid geometry holds true chiefly for the largest network; in only a few smaller components were some cyclic structures observed within principally linear-dendritic patterns. It is notable that the cyclic structure associated with epidemic STD transmission reported elsewhere ${ }^{1}$ is non-planar (that is, too complex to depict the network in a plane without crossed lines), while the presently reported cyclic pattern, associated with moderate HIV transmission, is planar.

Evidence from case surveillance of gonorrhoea-the most common reportable STD during the early period-suggests that sexual behaviour among gay men in Colorado Springs was already changing. For the first six months of 1981, before the presence of what would eventually be called AIDS was formally announced, ${ }^{5} 16 \%$ of all male gonorrhoea cases occurred in gay men. This proportion declined in two steps: from 1982 to 1985, to about 5-6\% annually; and to about 1\% by 1987 . We suspect that the hybrid geometry noted in the largest component during the early part of the HIV epidemic reflects this stepwise apparent decrease in HIV risk behaviour among high risk gay men. Thus a geometry somewhere between epidemic and endemic transmission would be expected, presumably reflecting stepwise decrease in epidemicity.

The fragmented nature of components other than the largest one during the early period helps to explain the modest HIV/AIDS burden reported from Colorado Springs: the risk networks of susceptible individuals have been insufficiently cohesive to sustain substantial endogenous HIV transmission..$^{6-8}$ Such fragmentation is more likely to reflect epidemiological reality than methodological artefacts. While it is true that contact tracing data contain gaps, they have been shown by modelling 9 and empirically ${ }^{10}$ to be sufficient to approximate true network structure. Our contact tracing derived HIV risk networks accord not only with what is intuitively expected, but also with observed HIV secular trends. In addition, the conserved geometry of the largest component, even after removal of nearly half of its constituents (from 324 to 156 persons), gives us confidence in the reality of what we observe.

Nevertheless, several contact tracing outcomes argue for caution in interpretation of our risk network data: first, in the present study, only newly diagnosed HIV/AIDS cases were offered interview; second, not all of them were reached; third, cases usually identified a minority of exposed partners (as is common in gay populations at risk for STD/HIV ${ }^{11}$ ) because of forgetting ${ }^{12}$; and fourth, partners were often not located or precisely identified. The dearth of interviews of exogenously diagnosed HIV/AIDS patients is unlikely to have affected our results significantly because infection was presumably acquired elsewhere, and named partners would lead away from local risk networks. Although our health department has long enjoyed the trust of Colorado Springs gay communities, the national polemic surrounding HIV name reporting and contact tracing from the mid-1980s to the mid-1990s may have affected our patients' candour. As for military cases, they and their partners were seldom, in the early years, represented in the larger sexual network components. In addition, many military HIV/AIDS cases arrived in Colorado Springs as a consequence of the repatriation policy of the US Armed Forces, and were not apparently involved in endogenous HIV acquisition or transmission. It is thus unlikely that partial absence of linked data from the low military caseload markedly affected our picture of local sexual networks.

The "true" picture of our HIV sexual networks, particularly that of the largest component, is likely to contain more cyclic 
structures than demonstrated. We hypothesise that, in addition to the four factors listed above, underascertainment of connections may be related to the relatively short (one year) interview interval used. Diseases such as AIDS tend to have long and uncertain latency periods; probabilistically, a longer interval may have permitted observation of historical connections tying previously unconnected cases into cycles-a phenomenon also likely to occur in relatively closed and small sexual networks such as those making up our largest component. In addition, it is possible that our HIV contact tracing efforts contributed to the fragmentation risk networks and the breaking up of cycles that are associated with enhanced network connectivity and enhanced transmission potential. ${ }^{10}$ Finally, the fact that contact tracing enabled us to identify the one risk network in Colorado Springs where, presumably, much (if not most) endogenous HIV transmission occurred in the early years of the epidemic is strong evidence for the epidemiological value of this intervention. ${ }^{10}{ }^{13}$

\section{Authors' affiliations}

J J Potterat, L Phillips-Plummer, S Q Muth, D E Woodhouse, T S Maldonado-Long, H P Zimmerman, J B Muth, STD/HIV Program, El Paso County Department of Health and Environment, Colorado Springs, Colorado, USA

R B Rothenberg, Department of Family and Preventive Medicine, Emory University School of Medicine, Atlanta, Georgia, USA

\section{REFERENCES}

1 Potterat JJ, Muth SQ, Rothenberg RB, et al. Sexual network structure as an indicator of epidemic phase. Sex Transm Infect 2002;77(suppl):i152-8.

2 Potterat JJ, Phillips L, Muth JB. Lying to military physicians about risk factors for HIV infections [letter] JAMA 1987;257:1727.

3 Batageli V, Mrvar A. PAJEK - program for large network analysis. Available at: http://vlado.fmf.uni-li.si/pub/networks/pajek/

4 Wylie JL, Jolly A. Patterns of chlamydia and gonorrhea infection in sexual networks in Manitoba, Canada. Sex Transm Dis 2001;28:14-24.

5 Centers for Disease Control. Pneumocystis pneumonia - Los Angeles. MMWR 1981;30:250-2.

6 Potterat JJ, Woodhouse DE, Rothenberg RB, et al. AIDS in Colorado Springs: is there an epidemic? AIDS 1993;7:1517-21.

7 Woodhouse DE, Rothenberg RB, Potterat JJ, et al. Mapping a social network of heterosexuals at high risk of HIV infection. AIDS 1994:8:1331-6.

8 Potterat JJ, Rothenberg RB, Muth SQ. Network structural dynamics and infectious disease propagation. Int J STD AIDS 1999;10:182-5.

9 Ghani AC, Donnelly CA, Garnett GP. Sampling biases and missing data in explorations of sexual partner networks for the spread of sexually transmitted diseases. Stat Med 1998;17:2079-97.

10 Rothenberg R, Sterk C, Toomey KE, et al. Using social network and ethnographic tools to evaluate syphilis transmission. Sex Transm Dis 1998;25:154-60.

11 Potterat JJ, Muth SQ, Muth JB. "Partner notification" early in the AIDS era: misconstruing contact tracers as bedroom police. In: Margolis E, ed. AIDS research/AIDS policy: competing paradigms of science and public policy, vol 6. Greenwich, CT: JAl Press, 1998:1-15.

12 Brewer DD, Garrett SB, Kulasingam S. Forgetting as a cause of incomplete reporting of sexual and drug injection partners. Sex Transm Dis 1999;26:166-76.

13 Potterat JJ. Contact tracing's price is not its value [editorial]. Sex Transm Dis 1997;24:519-21. 